\title{
Percutaneous Tennis Elbow Release Under Local Anaesthesia
}

\author{
M.A. Nazar, S. Lipscombe, S. Morapudi, G. Tuvo, R. Kebrle, W. Marlow and M. Waseem*
}

Macclesfield District General Hospital, Victoria Road, Macclesfield, Cheshire, SK10 3BL, UK

\begin{abstract}
Introduction: When the non-operative treatment of tennis elbow fails to improve the symptoms a surgical procedure can be performed. Many different techniques are available. The percutaneous release of the common extensor origin was first presented by Loose at a meeting in 1962. Despite the simplicity of the operation and its effectiveness in relieving pain with minimal scarring this procedure is still not widely accepted. This study presents the long-term results of percutaneous tennis elbow release in patients when conservative measures including local steroid injections have failed to relieve the symptoms.
\end{abstract}

Patients and Methods: Percutaneous release of the extensor origin was performed in 24 consecutive patients (seven male and seventeen female), providing 30 elbows for this study. The age of the patients ranged from 26 to 71 years with mean age of 55 years. The technique involved a day case procedure in the operating theatre using local anaesthesia without the need for a tourniquet. The lateral elbow was infiltrated with $5 \mathrm{mls} 1 \%$ lignocaine and $5 \mathrm{mls} 0.5 \%$ bupivicaine with 1:200,000 adrenaline. All operations were performed by the senior author. The patients were assessed post operatively by using DASH (disabilities of arm, shoulder and hand) score and Oxford elbow scores. The mean follow up period was 36 months (1-71months).

Results: Twenty one patients returned the DASH and Oxford elbow questionnaires. Four patients were lost in the follow up. The post operative outcome was good to excellent in most patients. Eighty seven percent of patients had complete pain relief. The mean post-op DASH score was 8.47 (range 0 to 42.9) and the mean Oxford elbow score was 42.8 (range 16 to 48). There were no complications reported. All the patients returned to their normal jobs, hobbies such as gardening, horse riding and playing musical instruments.

Conclusion: In our experience Percutaneous release of the epicondylar muscles for humeral epicondylitis has a high rate of success, is relatively simple to perform, is done as a day case procedure and has been without complications. Percutaneous release is a viable treatment option after failed conservative management of tennis elbow.

Keywords: Tennis elbow, percutaneous, release, tendinitis, Mill's Manipulation, local anaesthesia.

\section{INTRODUCTION}

In the English literature the term tennis elbow was first used in 1883 by Major in his paper Lawn-tennis elbow [1]. He defined it as a medical condition that causes pain on the lateral side of the elbow and is aggravated by wrist extension. The name is a misnomer because it rarely afflicts tennis players. According to Coonrad et al. [2] who assessed more than 1000 patients, fewer than $5 \%$ played golf or tennis. Nirschl [3] found that $50 \%$ of tennis players would experience symptoms related with lateral epicondylitis at least once in their life. Professional tennis players are less exposed to the risk of developing the disease due to better equipment and technique.

In $75 \%$ of cases, the dominant side is affected, suggesting that work-related forceful and repetitive wrist extension [1] may have a role in the pathogenesis. It has been suggested that most cases seen by the orthopaedic surgeon are the result of a work related "repetitive strain injury" [4]. Despite many theories having been proposed, the pathogenesis of this condition is still unclear. More than $90 \%$ of patients

*Address correspondence to this author at the Macclesfield District General Hospital, Victoria Road, Macclesfield, Cheshire, SK10 3BL, UK; Tel: 01625661315; Fax: 01625663153;

E-mails:was33uk@yahoo.com,was33@hotmail.com improve with non-operative management [2]. In a large series of 871 cases, Boyd [5] showed non-operative treatment was effective in 834 and that less than $5 \%$ required surgical treatment. Currently available treatment methods include acupuncture, ultrasonography, steroid injections, counterforce bracing, stretching exercises and crossfrictional massaging. Most of these treatments modalities have no scientific basis. The most successful non-operative treatment consists of avoidance of overuse counterforce bracing to relieve the insertion of the extensor tendons, steroid injection into the affected area and stretching exercises. Operative treatment is reserved for those who experience chronic symptoms of more than several months duration.

The operative treatment is effective in most of the patients regardless of the surgical technique. A review of current literature revealed that excellent or good results were achieved in $85-90 \%$ of cases [2-4]. The percutaneous release of the common extensor origin was first presented by Loose at a meeting in $1962[6,7]$. More than ten years later Baumgard [8], Yerger [9], and Powell [6] reported their experience with modifications of percutaneous techniques. Despite the simplicity of the operation and its effectiveness in relieving pain with minimal scarring this procedure is still not widely accepted. 
This study presents the long-term results of percutaneous tennis elbow release in patients when conservative measures including local steroid injections have failed to relieve the symptoms.

\section{MATERIALS AND METHODS}

All patients presenting with chronic lateral epicondylitis between 2003-2009 were eligible for the study. Percutaneous release was performed having fulfilled the following criteria:

1. Symptoms of two months or more duration following treatment.

2. Failed conservative management.

3. Localized pain over the lateral epicondyle.

4. Positive chair lift test.

5. No localized skin problems.

Cases were excluded if there had been previous surgery or other elbow pathology such as RA, OA, or radial tunnel syndrome.

Scoring was completed at the stated follow-up times with a single DASH and Oxford Elbow score performed in the outpatient clinic.

Ethics committee approval was not sought since this was a review study using a proven technique.

Conservative management included local steroid injection, physiotherapy, local ultrasound and stretching extensor exercises, and compression bracing. All patients were advised post-operatively to mobilise the wrist and the elbow several times a day and to repeat the following sequence: maintain the forearm in full pronation; fully extend the elbow; flex the wrist; fully; flex the fingers.

Patients were assessed post operatively by using DASH (disabilities of arm, shoulder and hand) score and Oxford elbow scores. Return to work and hobbies were also ascertained post-operatively.

\section{OPERATIVE TECHNIQUE}

The lateral elbow was infiltrated with $5 \mathrm{mls} 1 \%$ lignocaine and $5 \mathrm{mls} \quad 0.5 \%$ bupivicaine with $1: 200,000$ adrenaline. All patients had their operations performed by the senior author. The percutaneous release of the common extensor origin was performed in the operating theatre under local anaesthesia. The positioning for the procedure was supine. No tourniquet was used. A number 11 blade was inserted perpendicular to the skin anterior to the lateral epicondyle (Fig. 1). A skin incision one centimetre long was made. By moving the tip of the blade anteriorly and inferiorly from the lateral epicondyle a complete release of the common extensor origin was performed. A further displacement more distally of the common extensor tendon was then achieved by performing the Mill's manipulation. This manipulation consisted of a forcible, full extension of the elbow with the forearm fully pronated and the wrist and fingers held in flexion (Fig. 2). A gap of one centimetre, on average, was easily palpable between the lateral epicondyle and the retracted tendons at the end of the procedure. Skin closure was achieved with a single Steri-strip. A soft dressing was applied and early mobilisation was commenced. No wrist or elbow splints were recommended.

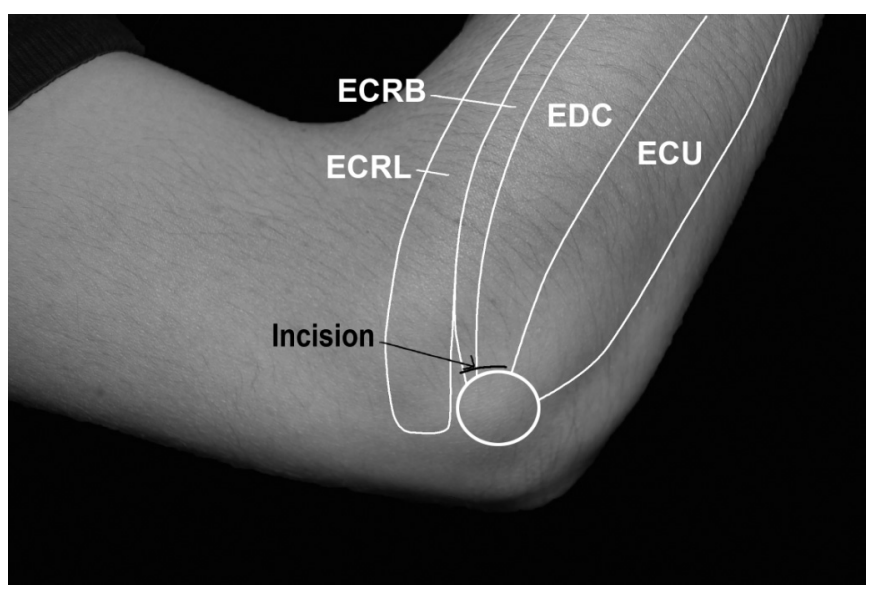

Fig. (1). Placement of skin incision.

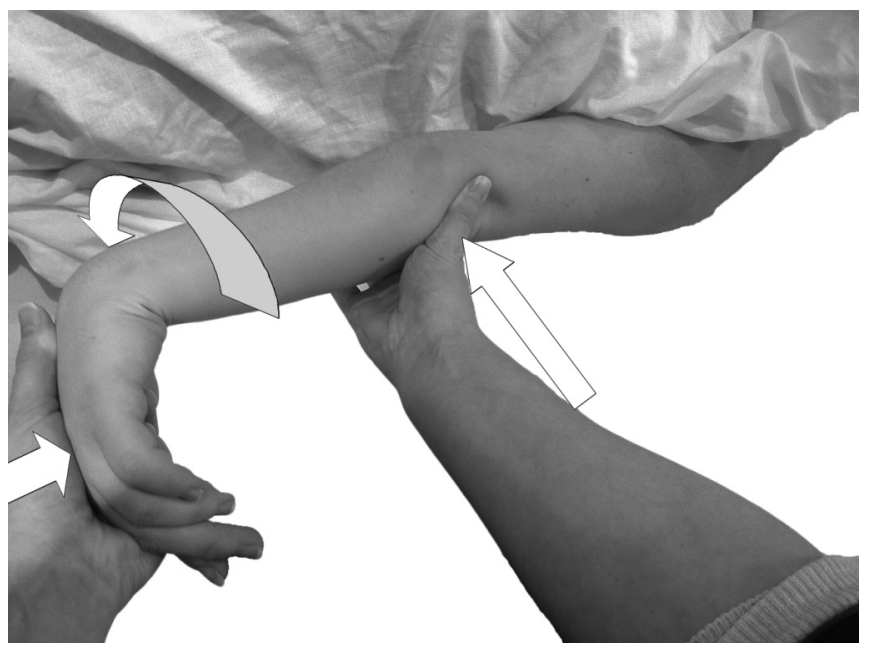

Fig. (2). Mill's Manipulation.

\section{RESULTS}

Over a period of 6 years (2003-2009), 30 elbows in 24 patients underwent percutaneous release. There were 7 male and 17 female patients in this series. The mean age at the time of operation was 53 years with a range of 26 to 71 years. In 14 patients ( $77 \%$ of cases), the dominant side was affected and interestingly, the dominant elbow was the side first affected in bilaterally affected cases. Four of the patients were sports enthusiasts (22\% of cases). Twelve patients' work $(66 \%$ of cases) involved heavy lifting or repetitive activities. The mean length of symptoms before presentation was 40 months (range 7 to 120 months). All patients had undergone previous conservative treatment, which failed to relieve their symptoms.

The follow up period was 1 to 71 months, with an average of 36 months. Four patients were lost to follow-up. The average time of return to work was 3 weeks ( 1 day to 12 weeks). The average time of reprise of sport activities was 14 weeks (min. 8, max 16 weeks). All patients were able to return to work performing preoperative tasks without any restriction. 
The post operative outcome was good to excellent in most patients. Eighty seven percent of patients had complete pain relief. The mean post-op DASH score was 8.47 (range 0 to 42.9 ) and the mean Oxford elbow score was 42.8 (range 16 to 48). No major complication occurred after surgery. More specifically, there were no cases of infection, complex regional pain syndrome or restriction in the range of motion of the elbow. One patient developed a wide haematoma on the lateral and dorsal aspect of the elbow, which extended into the proximal forearm. There was no need for treatment and it resulted in no infection or restriction in range of movement. All patients had a full range of motion at the elbow and all patients with excellent or good results returned to their former activities without any restriction. The scar was barely visible in most patients with no reports of loss of sensation or neuroma type pain around the scar.

\section{DISCUSSION}

According to the current literature, tennis elbow is treated successfully by non-operative measures in more then $90 \%$ of cases and in resistant cases a surgical treatment is available [2]. Several surgical options are available. Nirschl [10] suggests that 85 to $90 \%$ of cases can have symptomatic relief following any of a number of surgical options.

Percutaneous release of the origin of the common extensor tendons was proposed by Loose in 1962 [7] at the Hawkeye Sport Medicine Symposium, Iowa. No articles were published following this presentation. Baumgard [8] reported 35 cases of percutaneous release of tennis elbow in which an excellent result was achieved in 32 cases, while 3 cases were unsatisfactory. The surgical technique that was proposed is similar to our surgical technique. He performed the operation in the office or as an outpatient procedure in the office requiring infiltration of xylocaine. In our experience, infiltration of local anaesthetic in the area of surgery may alter the anatomical rapports and cloud the ability of the surgeon to appreciate the effectiveness of the procedure (palpation of the retraction of the extensors tendons). The axillary block provides a good control of the pain without compromising the procedure.

A similar technique was performed by Powell and Burke [6] and their results have been published more recently where they reviewed 20 patients at follow up from 5 to 36 months. They showed $85 \%$ excellent or good results. More recently Grundberg [11] presented the results of a percutaneous release of 32 cases of tennis elbow. The procedure was performed in the operating room under axillary block or general anaesthetic. He recommended using a Number 15 blade and his incision is placed transversely just distal to the lateral epicondyle. With a follow up period of an average of 26 months he found $90.6 \%$ of excellent and good results, but $22 \%$ of patients were not available for follow up and their information was" obtained entirely from charts".

Hohmann [12] in 1933 predated the described technique proposed by Nirschl [10], consisting in identification and excision of all pathological tissues at the common extensor tendon origin. This gave $97 \%$ improvement in symptoms and $85 \%$ of patients fully returned to work without pain. The results of an epicondylar stripping procedure for humeral epicondylitis with an open technique were reported on 26 cases of tennis elbow with $96 \%$ good or excellent results [13]. Garden [14] reported the results of 50 cases of lateral epicondylitis treated by Z-lengthening of the extensor carpi radialis at the wrist for which he stated, "Most obtained full and lasting relief'. This technique in the hands of other surgeons did not provide the same results. Carroll [15] a few years later only reported $20 \%$ of cases showed good results.

Wilhelm [16] recommended a complete denervation of the epicondylar region associated with decompression of the posterior interosseous nerve. He published the results of lateral epicondyle denervation alone (39 cases), associated with decompression of the posterior interosseous nerve (81 cases), and associated with isolated denervation of the supinator (46 cases). The results of the denervation were not improved by an additional radial nerve release. Excellent and good results were obtained in $90 \%$ of cases.

When non-operative treatments fail to improve the symptoms, a surgical procedure should be performed. Many different techniques are available. The percutaneous release of the common origin is a simple technique, can be performed under local anaesthetic with more comfort for the patient and leave a barely noticeable scar. The percutaneous release of the tennis elbow offers results that are comparable with all the more accredited procedures. A good selection of the patients should be carefully done, following the criteria outlined in the paper. A complete release of the common tendon of the extensors should be performed in order to obtain control of the symptoms.

Limitations of this study include the fact that it was retrospective and therefore there was no randomisation or control group. No pre-operative scores were available and only one scoring system was used.

\section{ACKNOWLEDGEMENT}

Declared none.

\section{CONFLICT OF INTEREST}

Declared none.

\section{REFERENCES}

[1] Major HP. Lawn-tennis elbow. BMJ 1883; 2: 557

[2] Coonrad RW, Hooper WR. Tennis elbow: its course, natural history, conservative and surgical management. J Bone Joint Surg Am 1973; 55(6): 1177-82.

[3] Nirschl RP. Tennis elbow. Orthop Clin North Am 1973; 4(3): 787800 .

[4] Boyer MI, Hastings H, 2nd. Lateral tennis elbow: "Is there any science out there?". J Shoulder Elbow Surg 1999; 8(5): 481-91.

[5] Boyd HB, McLeod AC Jr. Tennis elbow. J Bone Joint Surg Am 1973; 55(6): 1183-7.

[6] Powell SG, Burke AL. Surgical and therapeutic management of tennis elbow: An update. J Hand Ther 1991; 4: 64-68.

[7] Loose R. Tennis elbow: Twenty years experience. Presented at the Hawkeye Sports Medicine Symposium, Iowa City, Iowa 1984; pp. $5-7$.

[8] Baumgard SH, Schwartz DR. Percutaneous release of the epicondylar muscles for humeral epicondylitis. Am J Sports Med 1982; 10(4): 233-6.

[9] Yerger B, Turner T. Percutaneous extensor tenotomy for chronic tennis elbow: an office procedure. Orthopedics 1985; 8(10): 12613. 
[10] Nirschl RP. Muscle and tendon trauma: Tennis elbow. In: Morrey BF, Ed. The elbow and its disorders. Philadelphia: WB Saunders 1985; pp. 481-96.

[11] Grundberg AB, Dobson JF. Percutaneous release of the common extensor origin for tennis elbow. Clin Orthop Relat Res 2000; (376): $137-40$

[12] Hohmann G. Das wesen und die behandlung des sogenannten tennisellenbogens. Munch Med Wschr 1933; 80: 250-4.
[13] Spencer GE Jr, Herndon CH. Surgical treatment of epicondylitis. J Bone Joint Surg Am 1953; 35-A(2): 421-4.

[14] Garden RS. Tennis elbow. J Bone Joint Surg 1961; 43B: 100-6.

[15] Carroll RE, Jorgensen EC. Evaluation of the Garden procedure for lateral epicondylitis. Clin Orthop Relat Res 1968; 60: 201-4.

[16] Wilhelm A. Tennis elbow: treatment of resistant cases by denervation. J Hand Surg Br 1996; 21(4): 523-33.

(C) Nazar et al.; Licensee Bentham Open.

This is an open access article licensed under the terms of the Creative Commons Attribution Non-Commercial License (http://creativecommons.org/licenses/by-nc/3.0/) which permits unrestricted, non-commercial use, distribution and reproduction in any medium, provided the work is properly cited. 